\title{
Eco-friendly Synthesis of 1-Aryl-8-methoxythiazolo $[2 ', 3$ ' : 3, 4] [1, 2, 4] triazino [5, 6-b] Indoles under Microwave IR-Radiations
}

\author{
RAVINDER SINGH \\ Department of Chemistry, Govt. College for Women, Sampla, Rohtak, India. \\ *Corresponding author E-mail: gahlawat.ravinder@gmail.com \\ http://dx.doi.org/10.13005/ojc/310162
}

(Received: November 25, 2014; Accepted: January 11, 2015)

\section{ABSTRACT}

The different 1-Aryl-8-methoxythiazolo [2', 3' : 3, 4] [1, 2, 4] triazino [5, 6-b] indoles are synthesized in high yield in shorter reaction time under microwave irradiations keeping in view their antihistaminic, antithyroid, antitubercular, antifungal \& antibacterial activities.

Key words: Indole, Microwave, Isatin, Heterocyclic.

\section{INTRODUCTION}

The indoles exhibiting antihistaminic, antithyroid, antitubercular, antifungal, antibacterial activities $^{1-3}$, anthelminitics, antidepressants, platelet aggregation inhibitors, antineoplastic, vulcanization accelerators, photographic sensitizers ${ }^{4-11}$ properties are already been synthesized by different method but they requires longer reaction time and tedious workup. ${ }^{12-21}$ Microwave assisted reactions are gaining much more importance in synthetic organic chemistry due to dramatic reduction in time from days to hours and hours to minutes or seconds. ${ }^{22-23}$
The present work reports the synthesis of 1-Aryl-8-methoxythiazolo [2', 3' : 3, 4] [1, 2, 4] triazino $[5,6-b]$ indoles in a multi-step preparation in high yield in shorter reaction time(Scheme-I). Our work started by reacting 6-methoxyisatin with thiosemicarbazide in Anhyd. ethanol under microwave irradiation at 560W for 5-minutes to give 6-Methoxyisatin-3-thiosemicarbanzone (I). After separation, the 6-Methoxyisatin-3thiosemicarbanzone $(I)$ reacts with $p$ chlorophenacyl bromide under microwave irradiation at $560 \mathrm{~W}$ for 5 -minutes to give 6 Methoxyisatin-3-(4-p-chloropheny1-2-thiazoly1) hydrazone hydrobromide $\left(\mathrm{Ila} ; \mathrm{Ar}=\mathrm{p}-\mathrm{C}_{1} \mathrm{C}_{6} \mathrm{H}_{4}\right)(\mathrm{See}$ Scheme-1). 
<smiles></smiles>

Scheme-1<smiles>[R]/N=C1/C(=O)Nc2c1ccc(OC)c2[AlH]</smiles>

$\left[\mathrm{Ar}=\mathrm{p}-\mathrm{ClC}_{6} \mathrm{H}_{4^{-}}, \mathrm{p}-\mathrm{BrC}_{6} \mathrm{H}_{4^{-}},-\mathrm{C}_{6} \mathrm{H}_{4^{-}}\right]$
Similarly,

6-Methoxyisatin-3thiosemicarbazone (I) was also irradiated with $p$ bromophenacyl bromide, phenacyl bromide, under microwave irradiation at $560 \mathrm{~W}$ for 5 -minutes to give 6-Methoxyisatin-3-(4-p-bromopheny1-2-thiazoly1) hydrazone hydrobromide (Illb, $\mathrm{Ar}=\mathrm{p}-\mathrm{Br}_{6} \mathrm{H}_{4}$ ) and 6-Methoxyisatin-3-(pheny1-2-thiazoly1) hydrazone hydrobromide (Ilc; $\mathrm{Ar}=\mathrm{C}_{6} \mathrm{H}_{5}$ ) respectively. The results are shown in Table-1.

Table 1: 6-Methoxyisatin-3-(aryl-2-thiazoly1) hydrazone hydrobromide

\begin{tabular}{lcccc}
\hline S. NO. & Substrate(R) & Time(in minutes) & Yield(\%) & m.p. $\left({ }^{\circ} \mathrm{C}\right)$ \\
\hline 1. & $-\mathrm{pClC}_{6} \mathrm{H}_{4}-(\mathrm{Ila})$ & 5 & 94 & $>250^{\circ} \mathrm{C}$ \\
2. & $-\mathrm{pBrC}_{6} \mathrm{H}_{4}-(\mathrm{Ilb})$ & 5 & 92 & $>250^{\circ} \mathrm{C}$ \\
3. & $-\mathrm{C}_{6} \mathrm{H}_{5}(\mathrm{IIC})$ & 5 & 97 & $>250^{\circ} \mathrm{C}$ \\
\hline
\end{tabular}

We further explore our work by irradiated 6-Methoxyisatin-3-(4-p-chloropheny1-2-thiazoly1) hydrazone hydrobromide (Ila; $\mathrm{Ar}=\mathrm{p}-\mathrm{C}_{1} \mathrm{C}_{6} \mathrm{H}_{4}$ ) in a $\mathrm{POCl}_{3}$ under microwave irradiation at $560 \mathrm{~W}$ for 5- minutes to give 1-(p-Chlorophenyl)-8methoxythiazolo [2', 3' : 3, 4] [1, 2, 4] triazino [5, 6-b] indole (IIla, $\mathrm{R}=\mathrm{C} 1$ ). (See Scheme-2).<smiles>[R]N=C1C(=O)Nc2cc(OC)ccc21</smiles>

$\left[\mathrm{Ar}=\mathrm{p}-\mathrm{ClC}_{6} \mathrm{H}_{4^{-}}, \mathrm{p}-\mathrm{BrC}_{6} \mathrm{H}_{4^{-}},-\mathrm{C}_{6} \mathrm{H}_{4^{-}}\right]$

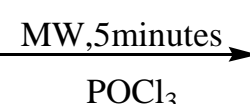

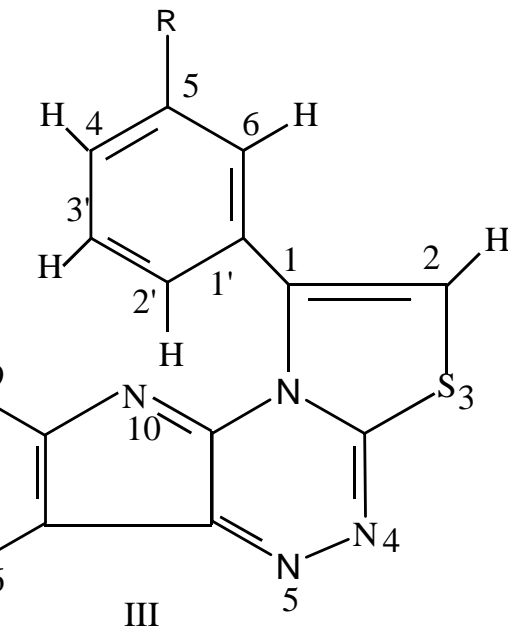

Scheme-2

$$
[\mathrm{R}=-\mathrm{Cl}, \mathrm{Br},-\mathrm{H}]
$$


Similarly, 6-Methoxyisatin-3-(4-pbromopheny1-2-thiazoly1) hydrazone hydrobromide (Illb; $\mathrm{Ar}=\mathrm{p}-\mathrm{BrC}_{6} \mathrm{H}_{4}$ ) and 6Methoxyisatin-3-(pheny1-2-thiazoly1) hydrazone hydrobromide (Ila; $\left.\mathrm{Ar}=\mathrm{p}-\mathrm{C}_{1} \mathrm{C}_{6} \mathrm{H}_{4}\right)\left(\mathrm{Ilc} ; \mathrm{Ar}=\mathrm{C}_{6} \mathrm{H}_{5}\right.$ ) were also irradiated in a mixture of $\mathrm{POCl}_{3}$ under microwave irradiation at $560 \mathrm{~W}$ for 5 -minutes to give 1-(p-bromophenyl)-8-methoxythiazolo [2', 3' : 3, 4] $[1,2,4]$ triazino $[5,6-\mathrm{b}]$ indole $(\mathrm{IIlb}, \mathrm{R}=\mathrm{Br})$ and 1 (phenyl)-8-methoxythiazolo [2', 3' : 3, 4] [1, 2, 4] triazino [5, 6-b] indole (IIlc, $R=H$ ) respectively. The results are shown in Table- 2 .

Table 2: 1-(p-Chlorophenyl)-8-methoxythiazolo

$[2 ', 3 ': 3,4][1,2,4]$ triazino $[5,6-b]$ indole

\begin{tabular}{lcccc}
\hline S. NO. & Substrate(R) & Time(in minutes) & Yield(\%) & m.p.( $\left.{ }^{\circ} \mathrm{C}\right)$ \\
\hline 1. & $-\mathrm{Cl}(\mathrm{III} \mathrm{a})$ & 5 & 89 & $>250^{\circ} \mathrm{C}$ \\
2. & $-\mathrm{Br}(\mathrm{IIIb})$ & 5 & 86 & $>250^{\circ} \mathrm{C}$ \\
3. & $-\mathrm{H}(\mathrm{IIIC})$ & 5 & 93 & $>250^{\circ} \mathrm{C}$ \\
\hline
\end{tabular}

\section{EXPERIMENTAL}

All the melting points reported are uncorrected. Infrared spectra $\left(\mathrm{n}_{\max }\right.$ in $\left.\mathrm{cm}^{-1}\right)$ were recorded in nujol mull or $\mathrm{KBr}$ on a Perkin-Elmer 842/Beckman IR-20 / Hitachi 215 spectrometers. The proton magnetic resonance spectra were recorded on a VXR-200 MHz or R-32 Perkin-Elmer $90 \mathrm{MHz}$ spectrometer in $\mathrm{CDC1}_{3}$ or $\mathrm{DMSO}-\mathrm{d}_{6}$ using tetramethylsilane (TMS) as internal reference stadnard. The chemical shifts are expressed in (ppm) units downfield from TMS. Mass spectra were scanned on a Jeol JMX-DX-300 spectrometer operating at $70 \mathrm{eV}$. Carbon, hydrogen and nitrogen analyses were carried out on a Yanaco MT-3 (JAPAN) instrument. Thin layer chromatography (TLC) were performed on silica-gel plates using acetone-benzene $(1: 3$ or $1: 2)$ as solvent system and iodine chamber as visualizing agent.

Typical procedure for the synthesis of 6Methoxyisatin-3-thiosemicarbanzone(l)

A mixture of 6-methoxyisatin $(0.18 \mathrm{~g}, 0.001$ $\mathrm{mol}$ ) in Anhyd. ethanol (2ml) and thiosemicarbazide $(0.1 \mathrm{~g}, 0.0011 \mathrm{~mol})$ in a mixture of water $(2 \mathrm{ml})$ and glacial acetic acid $(0.5 \mathrm{ml})$ was irradiated under microwave irradiation at $560 \mathrm{~W}$ for 5 -minutes. A yellow coloured solid formed during irradiation. The solid was filtered, washed well with water and crystallized from ethanol-DMF furnishing yellow crystals. Yield $0.247 \mathrm{~g}(95 \%)$, m.p. $265^{\circ} \mathrm{C}$. [Found : $\mathrm{N}, 22.68, \mathrm{~S}, 12.62 . \mathrm{C}_{10} \mathrm{H}_{10} \mathrm{~N}_{4} \mathrm{O}_{2} \mathrm{~S}$ requires $\mathrm{N}, 22.40$;
S, 12.80\%]; IR: 825, 860 (1, 2, 4-trisubstituted benzene ring), $1115(\mathrm{C}=\mathrm{S}), 1125$ \& 1370 (C-O-C stretching), $1620(\mathrm{C}=\mathrm{N}), 1700(\mathrm{C}=\mathrm{O}), 3200,3280$, $3400\left(\mathrm{NH}, \mathrm{NH}_{2}\right)$.

Typical procedure for the synthesis of 6Methoxyisatin-3-(4-p-chloropheny1-2-thiazoly1) hydrazone hydrobromide (Ila, $\left.\mathrm{Ar}=\mathrm{p}-\mathrm{C} 1 \mathrm{C}_{6} \mathrm{H}_{4}{ }^{-}\right)$

6-Methoxyisatin-3-thiosemicarbazone (I, 0.250g, $0.001 \mathrm{~mole})$ and $\mathrm{p}$-cholorophenacyl bromide $(0.234$ $\mathrm{g}, 0.001 \mathrm{~mol})$ in DMF $(6 \mathrm{ml})$ was irradiated under microwave irradiation at 560W for 5-minutes, and poured into ice-water. The solid thus separated, was filtered, washed with water and crystallized from aq. DMF to give lla as yellow crystals, yield $0.437 \mathrm{~g}$ (94\%), m.p. $>250^{\circ} \mathrm{C}$ [Found: N, 12.20; S, 6.90. $\mathrm{C}_{18} \mathrm{H}_{14} \mathrm{~N}_{4} \mathrm{O}_{2} \mathrm{SC} 1 \mathrm{Br}$ requires $\mathrm{N}, 12.03 ; \mathrm{S}, 6.87 \%$ ]; IR : 810, 870 (1, 2, 4-trisubstituted benzene ring), 1150, 1370 (C-0-C stretching), $1630(\mathrm{C}=\mathrm{N}), 1690$ $(\mathrm{C}=0), 3180,3300$ (N-H stretching).

Following members of the series were also prepared in a similar way

Illb $\left(\mathrm{Ar}=\mathrm{p}-\mathrm{BrC}_{6} \mathrm{H}_{4}^{-}\right)$: yield 0.469g (92\%), m.p. $>250^{\circ}$ [Found: N, $11.16 \mathrm{~S}$, 6.42. $\mathrm{C}_{18} \mathrm{H}_{14} \mathrm{~N}_{4} \mathrm{O}_{2} \mathrm{SBr}_{2}$ requires $\mathrm{N}, 10.98 ; \mathrm{S}, 6.27 \%$; IR: 1535 (C-N stretching), $1610(\mathrm{C}=\mathrm{N}), 1685(\mathrm{C}=0)$, 3170 (N-H stretching).

Ilc $\left(\mathrm{Ar}=\mathrm{C}_{6} \mathrm{H}_{5}\right)$ : yield $0.418 \mathrm{~g}(97 \%)$, m.p. > $250^{\circ}$ [Found: N, 14.54; S, 8.47. $\mathrm{C}_{18} \mathrm{H}_{15} \mathrm{~N}_{4} \mathrm{O}_{2} \mathrm{SBr}$ 
requires N, 14.49; S, 8.27\%]; IR: 1515 (C-N stretching), $1610(\mathrm{C}=\mathrm{N}), 1690(\mathrm{C}=0), 3180(\mathrm{~N}-\mathrm{H}$ stretching).

Typical procedure for the synthesis of 1-(pChlorophenyl)-8-methoxythiazolo [2', 3' : 3, 4] [1, 2 , 4] triazino [5, 6-b] indole (IIla, $\mathrm{R}=\mathrm{C} 1$ )

Compound Ila $(0.465 \mathrm{~g}, 0.001 \mathrm{~mole})$ in $\mathrm{POC}_{3}(4 \mathrm{ml})$ was irradiated under microwave irradiation at $560 \mathrm{~W}$ for 5 -minutes. The reaction mixture was poured into water and neutralised with aq. $\mathrm{K}_{2} \mathrm{CO}_{3}$ solution. The solid, thus separated, was filtered, washed well with water and crystallised from aq. DMF to furnish Illa as yellow crystals, yield $0.325 \mathrm{~g}(89 \%)$, m.p. $>250^{\circ}$ [Found: C, 59.10 ; H, 3.12; $\mathrm{N}, 15.38 ; \mathrm{S}, 8.55 . \mathrm{C}_{18} \mathrm{H}_{11} \mathrm{~N}_{4}$ OSC1 requires $\mathrm{C}$, 58.93; H, 3.01; N, 15.27; S, 8.73\%]; IR : 1575 (C-N), 1610 $(\mathrm{C}=\mathrm{N})$; PMR (DMSO-d): d3.95 (3H, s, $\left.\mathrm{C}_{8}-\mathrm{OCH}_{3}\right) ; 7.95$ $\left(1 \mathrm{H}, \mathrm{s}, \mathrm{C}_{2}-\mathrm{H}\right) ; 6.4-8.4(7 \mathrm{H}, \mathrm{m}, \mathrm{ArH})$.
Following members of the series were also prepared in a similar way

IIlb $(\mathrm{R}=\mathrm{Br})$ : yield $0.353 \mathrm{~g}(86 \%)$, m.p. > $250^{\circ}$ [Found : C, 52.76; H, 2.80; N, 13.87; S, 7.84. $\mathrm{C}_{18} \mathrm{H}_{11} \mathrm{~N}_{4} \mathrm{OSBr}$ requires $\mathrm{C}, 52.55 ; \mathrm{H}, 2.67 ; \mathrm{N}$, 13.62; S, 7.78\%]; IR: 1570 (C-N stretching), 1600, $1610(\mathrm{C}=\mathrm{N})$.

Illc $(R=H)$ : yield $0.308 g(93 \%)$, m.p. > $250^{\circ}$ [Found : C, 65.21; H, 3.74; N, 16.66; S, 9.52. $\mathrm{C}_{18} \mathrm{H}_{12} \mathrm{~N}_{4} \mathrm{OS}$ requires $\mathrm{C}, 65.06 ; \mathrm{H}, 3.61 ; \mathrm{N}, 16.86$; S, 9.63\%]; IR: 1570 (C-N stretching), 1600, 1620 $(\mathrm{C}=\mathrm{N})$.

\section{ACKNOWLEDGMENTS}

We thank Professor D. Villemin (France), Dr. R. Sharma (Dayton, USA) and Professor A.J. Bellamy (Swindon, UK) for inspiration.

\section{REFERENCES}

1. Mohan, J.; Anjaneyulu, G.S.R.; Kiran, Indian J. Chem. 1988, 27B, 128.

2. Snyder, H.R.; Benjamin, L.E., J. Med. Chem., 1966, 9, 402.

3. Pharmacal, N.; Neth. Appl. 1964, 6, 380 ; Chem. Abstr., 1965, 62,2780.

4. Trepanier, D.L.; Krieger, P.E., U.S. Pat., 1972, 3, 641; Chem. Abstr., 1972, 76, 127024k.

5. Sharpe, C.J.; Shadbolt, R.S.; Ashford, A.; Ross, J.W.; J. Med. Chem., 1971, 14, 977.

6. Kochhar, M.M.; Williams, B.B.; J. Med. Chem., 1972, 15, 322.

7. Kano, S.; Noguchi, T.; Japan Pat., 1971, 71, 836; Chem. Abstr., 1972, 76, 25295g.

8. Renfrew, E.E.; Pons, H.W.; U.S. Pat., 1976, 3, 130; Chem. Abstr., 1976, 85, 22753 e.

9. Jenkins, P.W.; Brooker, L.G.S.; U.S. Pat., 1972, 3, 81; Chem. Abstr., 1973, 78, 85949z.

10. Hinata, M.; SHiba, K.; Takei, H.; Sato, A.; Sakai, T.; Ger. Offen., 1974, 2, 418; Chem. Abstr., 1975, 82, 49815K.

11. Brooker, L.G.S.; U.S. Pat., 1937, 2,729; Chem. Abstr., 1937, 31, 6989.

12. (a) Romagnoli, R; Baraldi, P.G; Cruz-Lopez, C; Preti, D; Bermejo, J; Estavez, F.; Chem. Med. Chem., 2009, 4, 1668. (b) Bursavich, M.G.; Gilbert, A.M.; Lombardi, S; Georgiadis, K. E; Reifenberg, E, Flannery, C. R; Morris, E.A; Bio-org. Med. Chem. Lett. 2007, 17, 5630.

(c) Konkel, M.J; Packiarajan, M; Chem. H Topiwala, U.P.; Jimenez, H; Talisman, I.J; Coate, H; Walker, M.W.; Bioorg. Med. Chem. Lett, 2006, 16, 3950.

(d) Lam, P.Y.S; Vinoent, G; Clark, C.G; Dcudon, S; Jadhav, P.K.; Tetrahedron Lett.; 2001, 42, 3415.

13. (a) Shindikar, A.V; Khan, F;Viswanathan, C.L.; Eur. J. Med. Chem. 2006, 41, 786.

(b) Moser, P; Sallmann, A; Wieserberg, I.; J. Med. Chem. 1990, 33, 2358.

(c) Sarges, R; Howard, H. R. Koe. H.K; Weissman, A., J. Med. Chem. 1989, 32, 437.

14. Peet, N.; J. Heterocyclic Chem.; 1990, 17, 1514.

15. For reviews, see: (a) Chem, Y; Larock, R.C.; In Modern Arylation Methods, Ackerman, J.; ED Wiley/ VCH New York, 2009, 401.

(b) Sanz, R.;Org. Prep. Proced. Int.; 2008, 40, 215.

16. (a) Lin, Z; Larock, R. C.; J. Org. Chem. 2006, 71, 3198. 
(b) Lin, Z; Larock, R. C.; Org. Lett. 2003, 5, 4673. (c) Lin, Z; Larock, R. C.; Org. Lett. 2004, 6, 99.

17. Lin, Z; Larock, R. C.; J. American Chem. Soc.; 2005, 127, 13112.

18. Pintori, D. G; Greaney, M.F.; Org. Lett. 2010, 12, 168.

19. Yoshida, H; Shirakawa, E; Honda, Y; Hiyama, T.; Angew. Chem, Inted.; 2002, 41, 3246.
20. Zhao, J; Larock, R.C.; J.Org. Chem.; 2007, 72, 583.

21. Rogness D. C; Larock, R.C.; Tetrahedron Lett.; 2009, 50, 4003.

22. Lin, Z; Larock, R.C.; J. Org. Chem.; 2006, 71, 3198.

23. Peddibhotla, S.; Curr. Bioact. Compd.; 2009, 5, 20. 